\title{
Restratification of Abyssal Mixing Layers by Submesoscale Baroclinic Eddies
}

\author{
JÖRN CALLIES \\ California Institute of Technology, Pasadena, California
}

(Manuscript received 20 April 2018, in final form 12 July 2018)

\begin{abstract}
For small-scale turbulence to achieve water mass transformation and thus affect the large-scale overturning circulation, it must occur in stratified water. Observations show that abyssal turbulence is strongly enhanced in the bottom few hundred meters in regions with rough topography, and it is thought that these abyssal mixing layers are crucial for closing and shaping the overturning circulation. If it were left unopposed, however, bottom-intensified turbulence would mix away the observed mixing-layer stratification over the course of a few years. It is proposed here that the homogenizing tendency of mixing may be balanced by baroclinic restratification. It is shown that bottom-intensified mixing, if it occurs on a large-scale topographic slope such as a midocean ridge flank, not only erodes stratification but also tilts isopycnals in the bottom few hundred meters. This tilting of isopycnals generates a reservoir of potential energy that can be tapped into by submesoscale baroclinic eddies. The eddies slide dense water under light water and thus restratify the mixing layer, similar to what happens in the surface mixed layer. This restratification is shown to be effective enough to balance the homogenizing tendency of mixing and to maintain the observed mixing-layer stratification. This suggests that submesoscale baroclinic eddies may play a crucial role in providing the stratification mixing can act on, thus allowing sustained water mass transformation. Through their restratification of abyssal mixing layers, submesoscale eddies may therefore directly affect the strength and structure of the abyssal overturning circulation.
\end{abstract}

\section{Introduction}

The return of Antarctic Bottom Water from the abyss back to the surface requires water mass transformation by diapycnal mixing (e.g., Lumpkin and Speer 2007; Talley 2013). While small-scale turbulence in the bulk of the abyssal ocean is relatively weak, turbulence levels are elevated where tidal or geostrophic flows pass over a rugged seafloor (e.g., Polzin et al. 1997; Ledwell et al. 2000; St. Laurent et al. 2012; Waterhouse et al. 2014). Internal waves are excited, become nonlinear, and break, typically within a few hundred meters of the ocean bottom (e.g., Legg and Huijts 2006; Garrett and Kunze 2007; Muller and Bühler 2009; Nikurashin and Ferrari 2010; Nikurashin and Legg 2011). This topographically induced turbulence can achieve substantial water mass transformation and thus help close the large-scale overturning circulation of the abyssal ocean, producing a few tens of Sverdrups $\left(1 \mathrm{~Sv} \equiv 10^{6} \mathrm{~m}^{3} \mathrm{~s}^{-1}\right)$ of both diapycnal up- and downwelling (e.g., St. Laurent et al. 2001; Jayne 2009; de Lavergne et al. 2016; Ferrari et al. 2016; McDougall and Ferrari 2017; de Lavergne et al. 2017; Holmes et al. 2018; Callies and Ferrari 2018b).

Corresponding author: Jörn Callies, jcallies@caltech.edu
For the topographically generated turbulence to have an effect on the overturning, however, the mixing must occur in stratified water. Otherwise, the turbulence would mix mixed fluid, and there would be no water mass transformation (cf. Armi 1978, 1979; Garrett 1979, 1990). Observations do suggest that abyssal mixing layers are stratified: hydrographic sections show isopycnals that only gently bend down within the bottom $500-1000 \mathrm{~m}$, for example, on the flanks of the Mid-Atlantic Ridge (Fig. 1). Vertical profiles show a stratification that is reduced in the bottom few hundred meters by only about a factor of 3 (Fig. 2a, blue curve). There must be a process that maintains the stratification in abyssal mixing layers against the homogenizing effect of the turbulence.

When turbulence occurs on large-scale topographic slopes that intersect the ambient stratification, like on the flanks of the Mid-Atlantic Ridge, cross-slope mean flows develop in response to the mixing (e.g., Phillips 1970; Wunsch 1970; Garrett et al. 1993). These mean flows have a restratifying tendency: they advect dense water up the slope at the bottom and light water down the slope above. As in Munk's (1966) balance, the turbulent buoyancy flux divergence is balanced by mean advection, except that the mean flow is in the cross-slope direction instead of the vertical. A dipolar cross-slope 

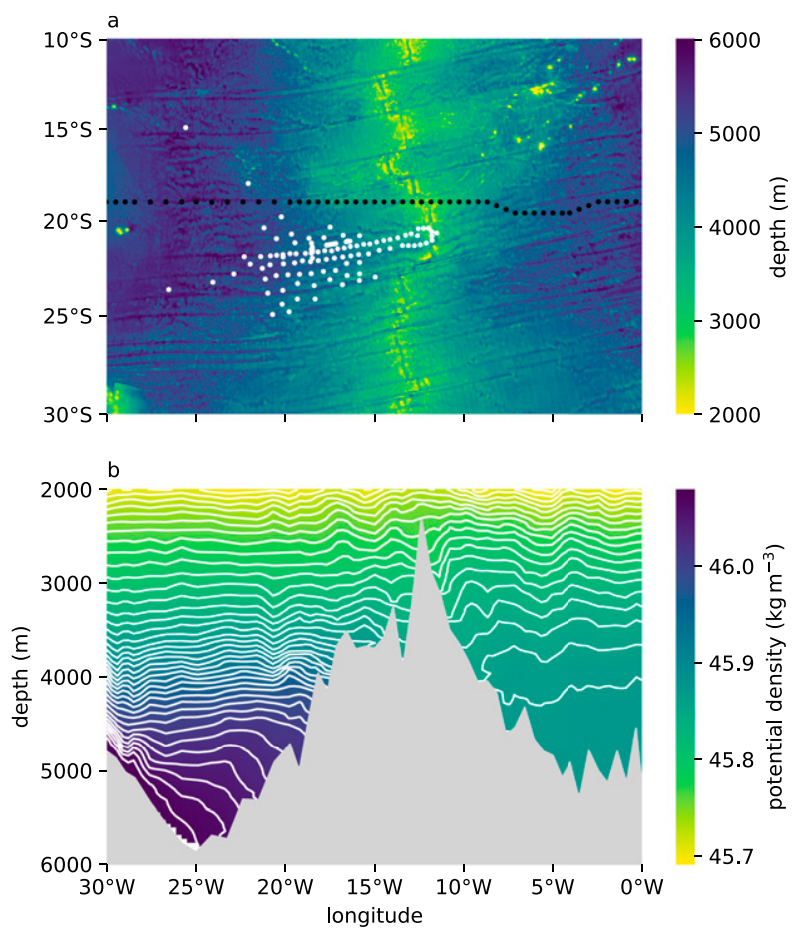

FIG. 1. South Atlantic Ridge bathymetry and observed hydrography. (a) Bathymetry from Smith and Sandwell (1997, updated), location of WOCE stations (black dots), and locations of Brazil Basin Tracer Release Experiment microstructure profiles (white dots); (b) WOCE section A09 of potential density referenced to 4000-m depth (1991, Research Vessel Meteor). Note the downward slope of isopycnals on the flanks of the Mid-Atlantic Ridge in the center of (b).

flow emerges because buoyancy fluxes have a pattern of divergence overlying convergence when mixing is bottom intensified.

While these cross-slope mean flows tend to increase the stratification in abyssal mixing layers, they are unlikely to be strong enough to maintain the observed stratification. As shown in section 3, the one-dimensional theory that describes this mechanism of mean-flow restratification, given realistically bottom-intensified mixing, produces mixing layers that are much more weakly stratified than what is observed (Fig. 2a, orange curve; Figs. 3a,b). Onedimensional theory is missing a crucial piece of physics.

Section 4 demonstrates that abyssal mixing-layer stratification can instead effectively be maintained by submesoscale baroclinic eddies. In Wenegrat et al. (2018), we showed that the steady state produced by one-dimensional theory is linearly unstable to baroclinic perturbations. The steady state's weak stratification implies strongly sloping isopycnals, a configuration that is associated with a large reservoir of potential energy available for release (Fig. 3b). Baroclinic instability taps into this potential energy reservoir by sliding dense water under light water in the crossslope direction at an instability scale of order $10 \mathrm{~km}$ and a growth rate of order $(10 \text { days })^{-1}$. The dynamics are similar to those of submesoscale baroclinic instabilities in the surface mixed layer (e.g., Boccaletti et al. 2007; Fox-Kemper et al. 2008; Callies et al. 2016).

Using three-dimensional calculations, I show in section 4 that this submesoscale baroclinic instability disrupts the one-dimensional balance of mixing layers on slopes by producing finite-amplitude eddies and substantial restratification. Over the course of a few months, baroclinic eddies eliminate the unrealistically weak mixinglayer stratification of the one-dimensional steady state (Fig. 2a, green curve; Fig. 3c). A new balance is achieved between the homogenizing effect of mixing and the restratifying effect of baroclinic eddies, in which the observed stratification is maintained (Fig. 2a, red curve; Fig. 3d). The restratification by submesoscale baroclinic eddies is much more powerful than that by mean flows and may be crucial in enabling topographically generated small-scale turbulence to mix stratified fluid, to incur substantial water mass transformation, and thus to affect the abyssal overturning circulation.

\section{Observational context}

To set the stage for the physical arguments presented in subsequent sections, I begin by reviewing observations from the microstructure component of the Brazil Basin Tracer Release Experiment (Polzin et al. 1997; St. Laurent et al. 2001). I focus on the profiles east of $27^{\circ} \mathrm{W}$ that are on the western flank of the Mid-Atlantic Ridge (Fig. 1a), where the bottom is rough and small-scale turbulence is enhanced. The bottom-intensified turbulence there is similar in magnitude and vertical structure to the turbulence observed over a rough seafloor elsewhere in the World Ocean (Waterhouse et al. 2014).

From the 1996 and 1997 cruises, the University of California, San Diego (UCSD), microstructure database (microstructure.ucsd.edu) holds 126 profiles for which a valid bottom depth is reported. For each profile, I calculate the stratification using the TEOS-10 seawater toolbox (McDougall and Barker 2011), and I average the stratification and the reported dissipation rates over 25-m bins of height above the bottom. I dismiss data within $100 \mathrm{~m}$ of the bottom because few profiles reach this deep, and averages are unreliable. I average across profiles and obtain $95 \%$ confidence intervals using the bootstrap percentile method (e.g., Efron and Hastie 2016). This yields a crude description of the bottom $2000 \mathrm{~m}$ on the western flank of the Mid-Atlantic Ridge, averaged over horizontal and temporal variations. This crudeness is acceptable here because the goal is not to match the observations exactly with the dynamical models discussed below, but to understand whether the proposed 

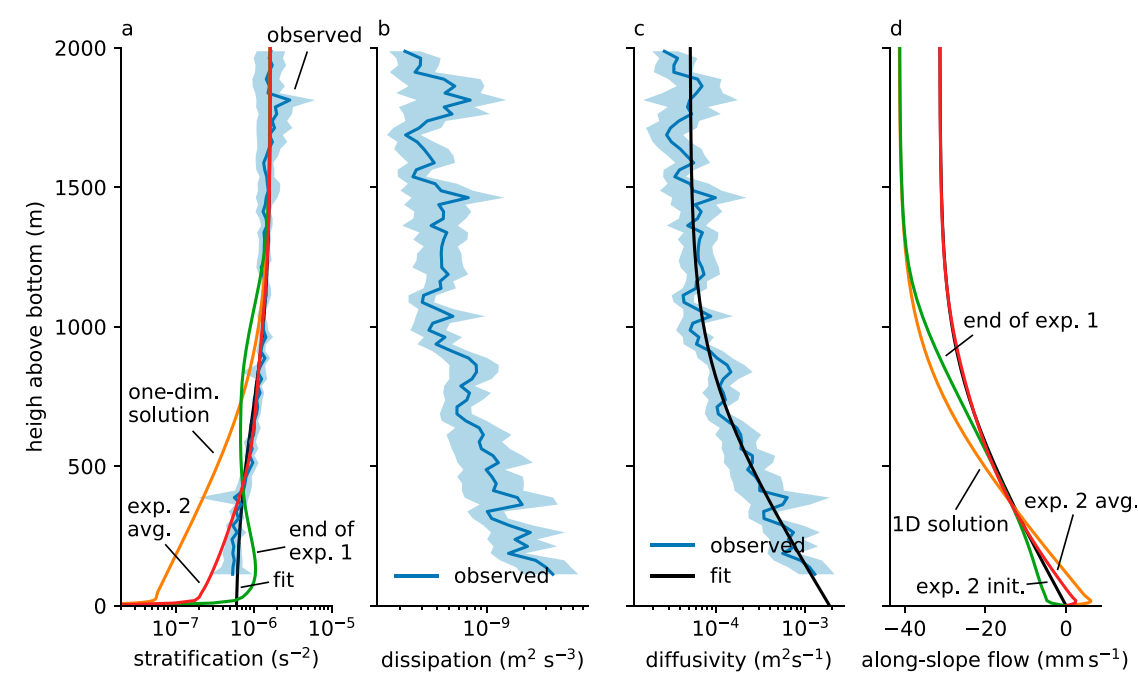

FIG. 2. Comparison between observations from the Brazil Basin Tracer Release Experiment microstructure profiles and the different models of the abyssal mixing layer considered in this paper. (a) The observed stratification is reduced in the bottom $800 \mathrm{~m}$; it is dramatically underpredicted by the one-dimensional solution, whereas baroclinic eddies effectively restore the mixing-layer stratification (experiment 1) and maintain it close to the observed value (experiment 2). (b) The dissipation rates inferred from microstructure profiles increase toward the bottom. (c) The turbulent diffusivity profile inferred from the observed dissipation rates and stratification is bottom enhanced by more than an order of magnitude; it is fit well by the functional form in (1). (d) The along-slope (i.e., along isopleth) flow is in approximate thermal wind balance with the buoyancy gradients associated with bending isopycnals (Figs. 1 and 3), except in a 20-m-thick inner boundary layer.

dynamics can predict the order of magnitudes of the observed profiles. See St. Laurent et al. (2001) for a comprehensive description of the data and their variability.

In a 1000-m-thick layer overlying the bottom, the stratification and dissipation rates show a notable change from relatively constant far-field values above (Fig. 2a, blue curve; Fig. 2b). The stratification decreases from a value of about $1.6 \times 10^{-6} \mathrm{~s}^{-2}$ at $1000-2000 \mathrm{~m}$ to about $5 \times 10^{-7} \mathrm{~s}^{-2}$ at $100 \mathrm{~m}$ (Fig. 2a, blue curve). The kinetic energy dissipation rate increases sharply from a value of about $4 \times 10^{-10} \mathrm{~m}^{2} \mathrm{~s}^{-3}$ at $1000-2000 \mathrm{~m}$ to about $3 \times 10^{-9} \mathrm{~m}^{2} \mathrm{~s}^{-3}$ at $100 \mathrm{~m}$ (Fig. 2b).

The observed dissipation rate $\varepsilon$ is commonly related to the vertical buoyancy flux by $F^{z}=-\Gamma \varepsilon$, where $\Gamma$ is an empirical nondimensional flux ratio typically assumed constant and equal to 0.2 (Osborn 1980). ${ }^{1}$ The buoyancy flux thus inferred from the dissipation rates increases in magnitude toward the bottom. Together with the decrease in stratification $B_{z}$, this gives rise to a sharp

\footnotetext{
${ }^{1}$ Variations in the flux ratio $\Gamma$ with stratification and dissipation rate have been detected in numerical simulations and laboratory experiments (e.g., Gregg et al. 2018). These effects are too small to affect the qualitative results of this paper and are neglected for simplicity.
}

increase in the turbulent diffusivity $\kappa=-F^{z} / B_{z}$ (Fig. 2c). Fitting the functional form

$$
\kappa=\kappa_{0}+\kappa_{1} e^{-z / h}
$$

yields a far-field diffusivity of $\kappa_{0}=5.2 \times 10^{-5} \mathrm{~m}^{2} \mathrm{~s}^{-1}$, a bottom enhancement of $\kappa_{1}=1.8 \times 10^{-3} \mathrm{~m}^{2} \mathrm{~s}^{-1}$, and a decay scale of $h=230 \mathrm{~m}$. I will use these values, together with the far-field buoyancy frequency $N=1.3 \times 10^{-3} \mathrm{~s}^{-1}$, throughout the paper (Table 1).

\section{One-dimensional dynamics: Mean-flow restratification}

In this section, I demonstrate that the observed stratification in the mixing layer cannot be reproduced by one-dimensional dynamics. I consider the dynamics of a rotating fluid adjacent to a planar slope that is inclined at an angle $\theta$ with respect to the horizontal, with the flow depending on the slope-normal coordinate only (e.g., Wunsch 1970; Phillips 1970; Thorpe 1987; Garrett et al. 1993). I assume the fluid to have a constant far-field buoyancy frequency $N$, subject it to the bottomintensified mixing inferred from the Brazil Basin observations (Fig. 2c), and consider the local response. If 

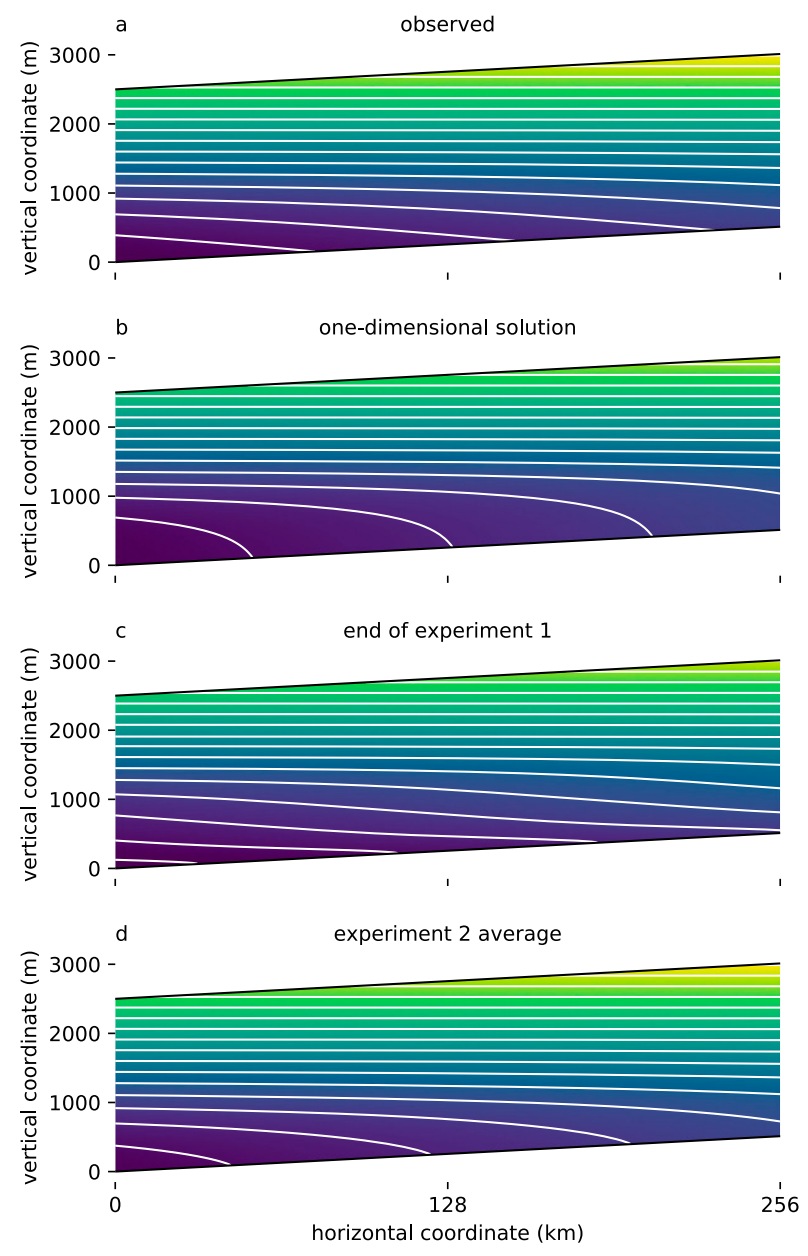

FIG. 3. Buoyancy sections $N^{2}(z \cos \theta+x \sin \theta)+\bar{b}$ in the crossslope-vertical plane: (a) as obtained by averaging the observed stratification profiles and fitting an analytical profile, (b) as predicted by steady one-dimensional dynamics and as used as initial conditions for experiment 1 , (c) as produced by baroclinic restratification in experiment $1(t=300$ days $)$, and (d) as produced by experiment 2 (time average over $t=3000-6000$ days), showing the maintenance of the observed stratification by baroclinic eddies. Both shading and contours show the buoyancy field; the contour interval is $2.5 \times 10^{-4} \mathrm{~m} \mathrm{~s}^{-2}$. Note the sloping domain used in all calculations and the reduced vertical exaggeration compared to Fig. 1.

mean-flow restratification were dominant, such a onedimensional model would be able to predict the observed stratification change over the bottom few hundred meters (Fig. 2a, blue curve). But while the thickness of the layer over which the stratification is modified from its far-field value is accurately predicted, the mixing-layer stratification is predicted to be much weaker than what is observed (Fig. 2a, orange curve).

A number of solutions to the one-dimensional dynamics on slopes are available, but no full steady solution has been derived for the realistic case at hand. There is a
TABLE 1. Parameters appropriate for the western flank of the Mid-Atlantic Ridge in the Brazil Basin. These parameters are used in all calculations presented in this paper.

\begin{tabular}{lc}
\hline \hline Inertial frequency $f$ & $-5.5 \times 10^{-5} \mathrm{~s}^{-1}$ \\
Far-field buoyancy frequency $N$ & $1.3 \times 10^{-3} \mathrm{~s}^{-1}$ \\
Slope angle $\theta$ & $2 \times 10^{-3}$ \\
Far-field diffusivity $\kappa_{0}$ & $5.2 \times 10^{-5} \mathrm{~m}^{2} \mathrm{~s}^{-1}$ \\
Bottom-enhancement of diffusivity $\kappa_{1}$ & $1.8 \times 10^{-3} \mathrm{~m}^{2} \mathrm{~s}^{-1}$ \\
Decay scale of diffusivity $h$ & $230 \mathrm{~m}$ \\
Prandtl number $\sigma$ & 1 \\
Hyperviscosity/hyperdiffusivity $\mu$ & $6.25 \times 10^{4} \mathrm{~m}^{4} \mathrm{~s}^{-1}$ \\
\hline
\end{tabular}

well-known analytical solution for constant mixing coefficients (Wunsch 1970; Thorpe 1987), but it is quite different in character from the case with bottomintensified mixing. Thompson and Johnson (1996) considered bottom-intensified mixing coefficients, but their transient solution did not reach steady state. Garrett (1991) derived an approximate analytical solution for the steady state, but his solution is valid only outside an inner layer that carries the upwelling part of the dipolar mean flow. I here extend Garrett's solution into the inner layer and thus obtain a uniformly valid approximate analytical solution. ${ }^{2,3}$

The dynamics are best described in a coordinate system aligned with the slope, with $x$ being the crossslope coordinate (increasing upslope), $y$ being the along-slope coordinate, and $z$ being the slopenormal coordinate. ${ }^{4}$ The local dynamics can be isolated by splitting the buoyancy field into a far-field stratification and an anomaly, $B=N^{2}(z \cos \theta+$ $x \sin \theta)+b$. Assuming that neither the buoyancy anomaly $b$ nor the flow varies in the plane of the slope, and that the flow is in steady state, reduces the Boussinesq equations to

\footnotetext{
${ }^{2}$ Garrett (1990) presented numerical solutions to the full bottomintensified case, but his setting $A=f \kappa_{e} \cot \theta$ (his notation) with a variable $\kappa_{e}$ was not justified and renders the solutions invalid.

${ }^{3}$ In Callies and Ferrari (2018b), we obtained steady solutions for bottom-intensified mixing, but these solutions depended on our choice of Rayleigh drag as a parameterization for friction. These dragcontrolled boundary layers were nothing more than stand-ins for the insufficiently understood dynamics of these layers, in order to study what implications the up- and downwelling in these layers have for the large-scale circulation. Here, I resort to a more conventional diffusive parameterization of turbulent momentum transport. While it is not clear whether the diffusive closure is appropriate for turbulence produced by internal wave breaking, its form as a flux divergence at least excludes momentum sinks in the interior of the fluid.

${ }^{4}$ For the small slope angles typical of the abyssal ocean, the slope-normal coordinate nearly coincides with the vertical, so there is no need to distinguish between the two coordinates in the analysis of observations (section 2).
} 


$$
\begin{aligned}
-f v \cos \theta & =b \sin \theta+\left(\nu u_{z}\right)_{z}, \\
f u \cos \theta & =\left(\nu v_{z}\right)_{z}, \\
u N^{2} \sin \theta & =\left[\kappa\left(N^{2} \cos \theta+b_{z}\right)\right]_{z},
\end{aligned}
$$

where $u$ is the cross-slope velocity, $v$ is the along-slope velocity, and $f$ is the inertial frequency (assumed constant). Turbulent momentum transfer is parameterized by a diffusive closure, and the turbulent viscosity $\nu=\sigma \kappa$ is assumed to have the same vertical profile as the turbulent diffusivity (i.e., the turbulent Prandtl number $\sigma$ is assumed constant). The slope-normal velocity vanishes, as required by continuity, and pressure can be inferred from the slope-normal momentum balance. Boundary conditions are that the flow and buoyancy flux vanish at the slope (i.e., $u=0, v=0$, and $N^{2} \cos \theta+b_{z}=0$ at $z=0$ ); decay conditions $u_{z} \rightarrow 0$, $v_{z} \rightarrow 0$, and $b_{z} \rightarrow 0$ are enforced for $z \rightarrow \infty$. As detailed by Garrett (1991), the system in (2)-(4) can be reduced to

$$
\begin{gathered}
\left(\nu \psi_{z z}\right)_{z z}+\left(\frac{f^{2} \cos ^{2} \theta}{\nu}+\frac{N^{2} \sin ^{2} \theta}{\kappa}\right) \psi \\
=N^{2} \cos \theta \sin \theta+\frac{f^{2} \cos ^{2} \theta}{\nu} \kappa_{0} \cot \theta,
\end{gathered}
$$

where the streamfunction $\psi$ is defined by $\psi_{z}=u$. The boundary conditions become $\psi=0$ and $\psi_{z}=0$ at $z=0$, and $\psi \rightarrow \kappa_{0} \cot \theta$ as $z \rightarrow \infty$ (Thorpe 1987).

This system is easily solved numerically by limiting the domain to a large but finite height $H=4000 \mathrm{~m}$, projecting the original set in (2)-(4) onto 2048 Chebyshev polynomials, and solving the resulting linear system (Burns et al. 2016). The solutions are insensitive to imposing the upperboundary conditions at a finite height as long as the domain is tall enough to fit the layer over which the background stratification is significantly modified.

An illuminating approximate analytical solution can be found if the decay scale of mixing $h$ is much larger than the thickness of a frictional inner layer (cf. Garrett 1991; Garrett et al. 1993). The thickness of the inner layer is given by (cf. Wunsch 1970; Thorpe 1987)

$q^{4}=\frac{1}{4\left(\nu_{0}+\nu_{1}\right)}\left(\frac{f^{2} \cos ^{2} \theta}{\nu_{0}+\nu_{1}}+\frac{N^{2} \sin ^{2} \theta}{\kappa_{0}+\kappa_{1}}\right)=\frac{f^{2} \cos ^{2} \theta(1+S \sigma)}{4\left(\nu_{0}+\nu_{1}\right)^{2}}$,

where $S=N^{2} \tan ^{2} \theta / f^{2}$ is the slope Burger number, $\nu_{0}=\sigma \kappa_{0}$, and $\nu_{1}=\sigma \kappa_{1}$. The scale $q^{-1}$ is the boundary layer thickness that would emerge if the mixing coefficients were constant and equal to their bottom values. This scale is equal to the thickness of a conventional Ekman layer modified by a factor $(1+S \sigma)^{-1 / 4}$, which arises from a combination of the effects of the slope and stratification.
For parameters typical of the abyssal ocean, the scale $q^{-1}$ is much smaller than the decay scale $h$. For the western flank of the Mid-Atlantic Ridge sampled by the Brazil Basin Tracer Release Experiment, $f=-5.5 \times 10^{-5} \mathrm{~s}^{-1}$ for a latitude of $22^{\circ} \mathrm{S}, N=1.3 \times 10^{-3} \mathrm{~s}^{-1}$, and the mean slope is approximately $\theta=2 \times 10^{-3}$ (Fig. 1, Table 1), which gives a slope Burger number of $S=2.2 \times 10^{-3}$. The Ekman layer thickness is thus only marginally modified if the Prandtl number is not very large. For $\sigma=1$ and mixing coefficients obtained from the observations (Fig. 2c, Table 1), the inner-layer scale is $q^{-1}=8.3 \mathrm{~m}$. With $h=230 \mathrm{~m}$, it is thus safe to assume that $q h \gg 1$ and to apply boundary layer theory (e.g., Bender and Orszag 1999).

Outside the inner layer, the solution varies on a scale $z \sim h$, and the first term in (5) can be neglected (Garrett 1991). A solution valid in the outer layer is thus

$$
\psi^{(o)}=\cot \theta\left(\kappa_{0}+\kappa_{1} e^{-z / h} \frac{S \sigma}{1+S \sigma}\right) .
$$

The outer-layer solution does not satisfy the boundary condition at $z=0$, however, so an inner layer is present there. The inner-layer adjustment $\psi^{(I)}$ is determined by the homogeneous version of (5), but since $\psi^{(I)}$ varies on a scale $z \sim q^{-1}$, the first term in (5) is now of leading order. Variations in mixing coefficients, on the other hand, are slight over the inner-layer scale and can be neglected, such that the coefficients become constant:

$$
\psi_{z z z z}^{(I)}+4 q^{4} \psi^{(I)}=0 .
$$

The inner-layer correction thus has the same structure as the solution for constant mixing coefficients (Wunsch 1970; Thorpe 1987), but the boundary conditions must be matched to the outer-layer solution. Enforcing $\psi^{(O)}+\psi^{(I)}=0$ and $\psi_{z}^{(I)}=0$ at $z=0$ (neglecting much smaller outer-layer contributions to $\psi_{z}$ ), as well as decay conditions as $z \rightarrow \infty$, yields

$$
\psi^{(I)}=-\left(\kappa_{0}+\kappa_{1} \frac{S \sigma}{1+S \sigma}\right) \cot \theta e^{-q z}(\cos q z+\sin q z) .
$$

The sum of (7) and (9) is a uniformly valid approximate solution to (5). ${ }^{5}$ Figure 4 illustrates this solution and compares it to the numerical solution. The approximation is excellent.

The analytical solution shows that the cross-slope transport integrated over the outer layer is $-\kappa_{1}$ $\cot \theta S \sigma /(1+S \sigma)$ and that the inner-layer transport

\footnotetext{
${ }^{5}$ Garrett (1991) noted that the outer-layer solution (7) allows the Prandtl number $\sigma$ to be a function of height. If $\sigma$ variations are much slower than $q^{-1}$, the boundary layer approach still works, and $\sigma$ in (9) is simply replaced by its value at $z=0$.
} 

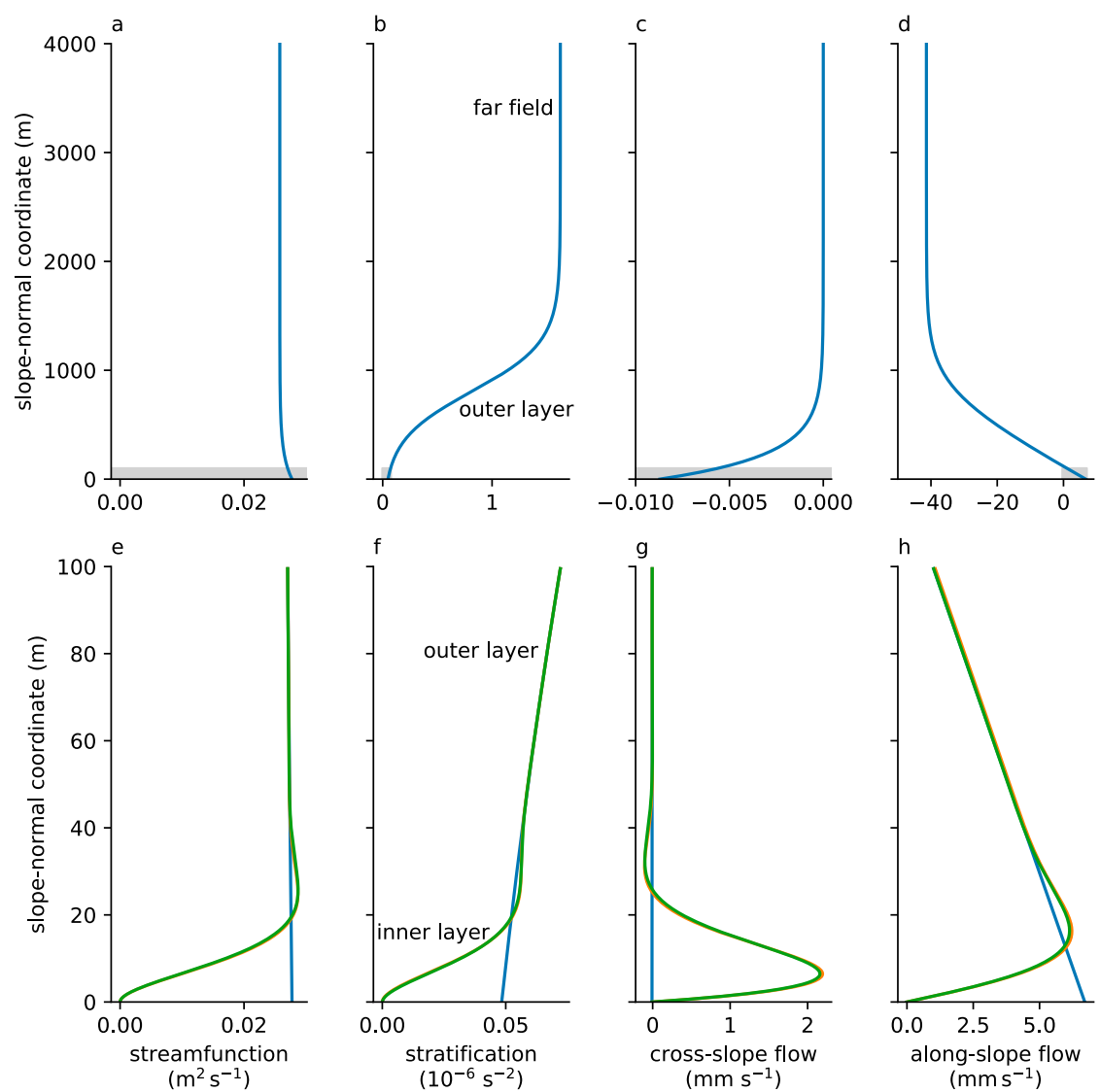

FIG. 4. One-dimensional steady state in the (top) outer layer and far field and (bottom) inner layer. Shown are the (a),(e) streamfunction, (b),(f) stratification, (c), (g) cross-slope flow, and (d),(h) along-slope flow as produced by the analytical solutions for the outer layer and far field (blue) and by the analytical solution including the inner-layer contribution (green). The approximate analytical solution matches the numerical solution (orange). Note the different scales of the abscissae in the upper and lower panels; the gray boxes in the upper panels show the plot range that is expanded in the lower layers, with the exception of $(\mathrm{g})$, which extends to much larger positive values than indicated in (c).

is $\kappa_{0} \cot \theta+\kappa_{1} \cot \theta S \sigma /(1+S \sigma)$. The solution thus exhibits compensating cross-slope transports of magnitude $\kappa_{1} \cot \theta S \sigma /(1+S \sigma)$ in the inner and outer layers and a net upslope transport $\kappa_{0} \cot \theta$ that is carried by the inner layer (cf. Thorpe 1987). The compensated part of the transport is the anticipated dipole of upwelling in the inner layer and downwelling in the outer layer that is due to the bottom intensification of mixing. Its magnitude, however, is reduced by a factor of $S \sigma /(1+S \sigma)$ compared to what it would be if the stratification in the outer layer was equal to the background. This factor is small for abyssal values if the Prandtl number is not large.

The stratification (in the slope-normal direction) follows directly from (7) and (9) through $\psi N^{2} \sin \theta=$ $\kappa\left(N^{2} \cos \theta+b_{z}\right)$ and is given by (Figs. $\left.4 \mathrm{~b}, \mathrm{f}\right)$

$$
N^{2} \cos \theta+b_{z}=N^{2} \cos \theta\left[\frac{\kappa_{0}}{\kappa_{0}+\kappa_{1} e^{-z / h}}+\frac{\kappa_{1} e^{-z / h}}{\kappa_{0}+\kappa_{1} e^{-z / h}} \frac{S \sigma}{1+S \sigma}-\left(\frac{\kappa_{0}}{\kappa_{0}+\kappa_{1}}+\frac{\kappa_{1}}{\kappa_{0}+\kappa_{1}} \frac{S \sigma}{1+S \sigma}\right) e^{-q z}(\cos q z+\sin q z)\right]
$$

The stratification is reduced in the outer layer up to $z \sim h \log \left(\kappa_{1} / \kappa_{0}\right)$, the level at which the diffusivity has decayed to approximately its background value. This reduction in the outer layer is strong if $\kappa_{0} \ll \kappa_{1}$ and 
$S \sigma /(1+S \sigma) \ll 1$. In the inner layer, the stratification decays from its reduced value at the inner edge of the outer layer to zero, as required to satisfy the boundary condition. The change in stratification in the inner layer has the same magnitude as the stratification value at the inner edge of the outer layer. There is a slight enhancement in stratification at the outer edge of the inner layer (around $z=25 \mathrm{~m}$ in Fig. 4f). For $\kappa_{0} \ll \kappa_{1}$ and $S \sigma /(1+S \sigma) \ll 1$, however, stratification changes over the inner layer are small compared to those in the outer layer (Figs. 4b,f), and the enhancement at the upper edge of the inner layer is too slight to reverse the horizontal buoyancy gradient $-b_{z} \sin \theta$ (cf. Phillips et al. 1986; Garrett et al. 1993).

The cross-slope flow implied by (7) and (9) is (Figs. 4c,g)

$$
\begin{aligned}
u= & -\frac{\kappa_{1} \cot \theta e^{-z / h}}{h} \frac{S \sigma}{1+S \sigma} \\
& +2 q \cot \theta\left(\kappa_{0}+\kappa_{1} \frac{S \sigma}{1+S \sigma}\right) e^{-q z} \sin q z
\end{aligned}
$$

This flow consists of a dipole of downslope flow in the outer layer and compensating upslope flow in the inner layer, plus an additional upslope flow in the inner layer that is proportional to $\kappa_{0}$. This uncompensated component of the inner-layer flow has the same functional form as if one had set $\kappa=\kappa_{0}$ uniformly (cf. Thorpe 1987), except that $q$ is now based on the enhanced bottom-mixing coefficients. The downslope flow in the outer layer decays with the same scale $h$ as the diffusivity, and its magnitude is proportional to $\kappa_{1} \cot \theta S \sigma /(1+S \sigma)$.

The along-slope shear implied by (7) and (9) is

$$
\begin{aligned}
v_{z}= & \frac{f \cot \theta \cos \theta}{\sigma}\left[\frac{\kappa_{1} e^{-z / h}}{\kappa_{0}+\kappa_{1} e^{-z / h}} \frac{S \sigma}{1+S \sigma}\right. \\
& \left.-\left(\frac{\kappa_{0}}{\kappa_{0}+\kappa_{1}}+\frac{\kappa_{1}}{\kappa_{0}+\kappa_{1}} \frac{S \sigma}{1+S \sigma}\right) e^{-q z}(\cos q z+\sin q z)\right]
\end{aligned}
$$

which can easily be integrated upward from $v=0$ at $z=0$ to infer the flow (Figs. 4d,h). There is significant shear in the outer layer, in the direction of Kelvin wave propagation, up to the height $z \sim h \log \left(\kappa_{1} / \kappa_{0}\right)$. This shear is in thermal wind balance with the horizontal buoyancy gradient $-b_{z} \sin \theta$ associated with the sloping isopycnals.

It should be noted that the solution derived above remains valid as $\kappa_{0} \rightarrow 0$, but it loses its confinement to the bottom: the reduced stratification and the alongslope shear extend to $z \rightarrow \infty$ (cf. Garrett 1990). This emphasizes that it is not the decay scale of mixing $h$ that sets the thickness of the layer over which the hydrography is modified, but the height at which the mixing coefficients stop changing, which occurs at $\kappa_{0} \sim \kappa_{1} e^{-z / h}$ or $z \sim h \log \left(\kappa_{1} / \kappa_{0}\right)$.

For Brazil Basin parameters, the one-dimensional solution produces a stratification that is much weaker than what is observed (Fig. 2a, blue and orange curves). The thickness of the outer layer $h \log \left(\kappa_{1} / \kappa_{0}\right)=800 \mathrm{~m}$ roughly matches the observed thickness of the layer with reduced stratification, but the reduction is much too severe. This shows that the cross-slope mean flow in (11) produced by one-dimensional dynamics, while displaying the anticipated dipolar structure, is much too weak to maintain realistic stratification. Instead of generating a strong dipole of cross-slope mean flow that balances the large diabatic buoyancy flux divergence and convergence produced by turbulence in a stratified fluid, one-dimensional dynamics predict a near homogenization of the mixing layer, in which the little water mass transformation that remains is balanced by weak crossslope mean flows. I will return to this point in section 5 .

\section{Three-dimensional dynamics: Baroclinic restratification}

The inability of the one-dimensional dynamics in (2)-(4) to reproduce the observed mixing-layer stratification (Fig. 2a, blue and orange curves) suggests that a crucial piece of physics is missing. In this section, I demonstrate that the one-dimensional balances are upset by a baroclinic instability and the resulting nonlinear baroclinic eddies. I consider two experiments in the same setup on a planar slope as in section 3, but I now allow three-dimensional and fully nonlinear flow. Experiment 1 starts from the one-dimensional steady state. This steady state is linearly unstable to baroclinic perturbations. The unstable modes grow, attain finite amplitude, and rapidly restratify the mixing layer, suggesting that we should not expect to see the one-dimensional steady state in observations. While experiment 1 clearly demonstrates the breakdown of the one-dimensional balances, it is a bit academic because the initial state has unrealistically weak stratification in the mixing layer, so the baroclinic restratification is unrealistically vigorous. But restratifying baroclinic eddies also emerge in the more realistic experiment 2 , which starts from a stratification close to what is observed in the Brazil Basin. Baroclinic eddies again develop, and their restratifying tendency effectively balances the homogenizing tendency of mixing.

\section{a. Baroclinic instability and restoration of stratification}

In Wenegrat et al. (2018), we showed that the onedimensional steady state obtained in section 3 is linearly 
unstable to baroclinic perturbations for parameters typical of abyssal mixing layers $\left(\kappa_{1} \gg \kappa_{0}\right.$ and $\left.q h \gg 1\right)$. The reduced stratification of the outer layer is associated with strongly sloping isopycnals and along-slope shear (Fig. 2d, orange curve; Fig. 3b), a configuration that allows counterpropagating Rossby waves to reinforce each other and grow. For completeness, I repeat the linear stability calculation here, using a domain height of $H=2500 \mathrm{~m}$, projecting the slope-normal structure onto 64 Chebyshev modes, and solving the resulting truncated eigenvalue problem (Burns et al. 2016). For the Brazil Basin parameters (Table 1), instability occurs over a range of along-slope wavenumbers, with a maximum growth rate of $(9 \text { days })^{-1}$ located at the along-slope wavenumber $l=5.2 \times 10^{-4} \mathrm{~m}^{-1}=2 \pi /(12 \mathrm{~km})$ (Fig. 5a). This most unstable perturbation grows through the release of available potential energy by buoyancy production in the bottom $400 \mathrm{~m}$, while the bottom Ekman layer exerts a significant drag (Fig. 5b). The most unstable mode displays the classic tilt of the cross-slope velocity into the shear (Fig. 5c; e.g., Pedlosky 1987; Vallis 2017) and the in-phase relationship between slope-normal velocity and buoyancy that allows potential energy release (Figs. 5d,e). The topographic slope does not stabilize the flow because it is much smaller than the isopycnal slope (Wenegrat et al. 2018; Fig. 3b).

To assess the restratification that is induced by the baroclinic instability and the finite-amplitude eddies that it produces, I now consider a fully nonlinear threedimensional numerical simulation of an abyssal mixing layer initialized with the one-dimensional steady state, using the same parameters as above (Table 1). Now allowing the flow to be time dependent and to vary in the plane of the slope (i.e., in $x$ and $y$ ), the Boussinesq equations in the slope-aligned coordinate system and with the hydrostatic approximation read

$$
\begin{aligned}
u_{t}+\mathbf{u} \cdot \nabla u-f v \cos \theta= & -p_{x}+b \sin \theta \\
& +\left(\nu u_{z}\right)_{z}+\mathcal{D} u, \\
v_{t}+\mathbf{u} \cdot \nabla v+f u \cos \theta= & -p_{y}+\left(\nu v_{z}\right)_{z}+\mathcal{D} v, \\
p_{z}= & b \cos \theta, \\
\nabla \cdot \mathbf{u}= & 0, \\
b_{t}+\mathbf{u} \cdot \nabla b+ & u N^{2} \sin \theta+w N^{2} \cos \theta \\
= & {\left[\kappa\left(N^{2} \cos \theta+b_{z}\right)\right]_{z}+\mathcal{D} b . }
\end{aligned}
$$

The hydrostatic approximation is justified if the aspect ratio of the flow $\delta$ is small (i.e., $\delta^{2} \ll 1$ ) and if $\delta \tan \theta \ll 1$, both of which are satisfied for the flows of interest here. In the equations above, u denotes the threedimensional velocity vector, $w$ is the slope-normal velocity component, $p$ is the pressure divided by a reference density, and $\mathcal{D}=-\mu\left(\partial_{x}^{4}+2 \partial_{x}^{2} \partial_{y}^{2}+\partial_{y}^{4}\right)$ is a hyperdiffusion operator that is added for numerical stability. I find the hyperviscosity/hyperdiffusivity $\mu=6.25 \times 10^{4} \mathrm{~m}^{4} \mathrm{~s}^{-1}$ to be large enough to suppress gridscale oscillations, albeit small enough to render negligible the energy loss due to hyperviscosity. The vertical boundary conditions are the same as for the one-dimensional equations (i.e., $u=0, v=0$, and $N^{2} \cos \theta+b_{z}=0$ at $z=0$ and $u_{z}=0, v_{z}=0$, and $b_{z}=0$ at $z=H$ ), augmented by no-normal-flow conditions (i.e., $w=0$ at $z=0$ and $z=H$ ), where $H=2500 \mathrm{~m}$ is again large enough to have a negligible effect on the flow in the mixing layer. In the plane of the slope, the domain is square with a side length of $L=256 \mathrm{~km}$, and periodic boundary conditions are imposed on velocities and buoyancy perturbations. This setup is similar to the widely used frontal-zone setup (e.g., Bretherton and Karweit 1975; Salmon 1980; Haidvogel and Held 1980; Taylor and Ferrari 2010; Callies and Ferrari 2018a) because it imposes a fixed domain-average cross-slope buoyancy gradient $N^{2} \sin \theta$. Note that in this setup on a slope, however, the mean buoyancy gradient in the true horizontal can and does change during restratification. Fourier bases with 256 modes each are used in $x$ and $y$, the $z$ direction is again projected onto 64 Chebyshev modes, and the system is fully dealiased. Time stepping is performed with a semi-implicit third-order four-stage Runge-Kutta scheme and a time step of $20 \mathrm{~min}$ (Burns et al. 2016).

I initialize the flow with the one-dimensional steady state (section 3), with small random perturbations (normally distributed with standard deviation $2 \times 10^{-7} \mathrm{~m} \mathrm{~s}^{-2}$ ) added to the buoyancy field to initiate the instability. As predicted by the linear calculation (Fig. 5a), the dominant baroclinic mode that emerges after a few $e$-folding times has an along-slope wavelength of about $12 \mathrm{~km}$. Around $t=50$ days, the instability has grown to an amplitude at which nonlinear terms become important. Secondary instabilities occur, and the flow becomes fully turbulent (Fig. 6). The dominant eddies grow in size because nonlinear-scale interactions preferentially transfer energy to larger scales and because restratification stabilizes the smaller scales. The eddies retain a scale somewhat smaller than the size of the domain, suggesting they are not constrained by the finite domain size.

The evolution of the flow can be tracked in the eddy kinetic energy budget:

$$
\begin{aligned}
K_{t}= & \left\langle\overline{u^{\prime} b^{\prime}} \sin \theta+\overline{w^{\prime} b^{\prime}} \cos \theta\right\rangle-\left\langle\bar{u}_{z} \overline{u^{\prime} w^{\prime}}+\bar{v}_{z} \overline{v^{\prime} w^{\prime}}\right\rangle \\
& -\left\langle\nu\left(\overline{u_{z}^{\prime 2}}+\overline{v_{z}^{\prime 2}}\right)\right\rangle+\left\langle\overline{u^{\prime} \mathcal{D} u^{\prime}}+\overline{v^{\prime} \mathcal{D} v^{\prime}}\right\rangle .
\end{aligned}
$$



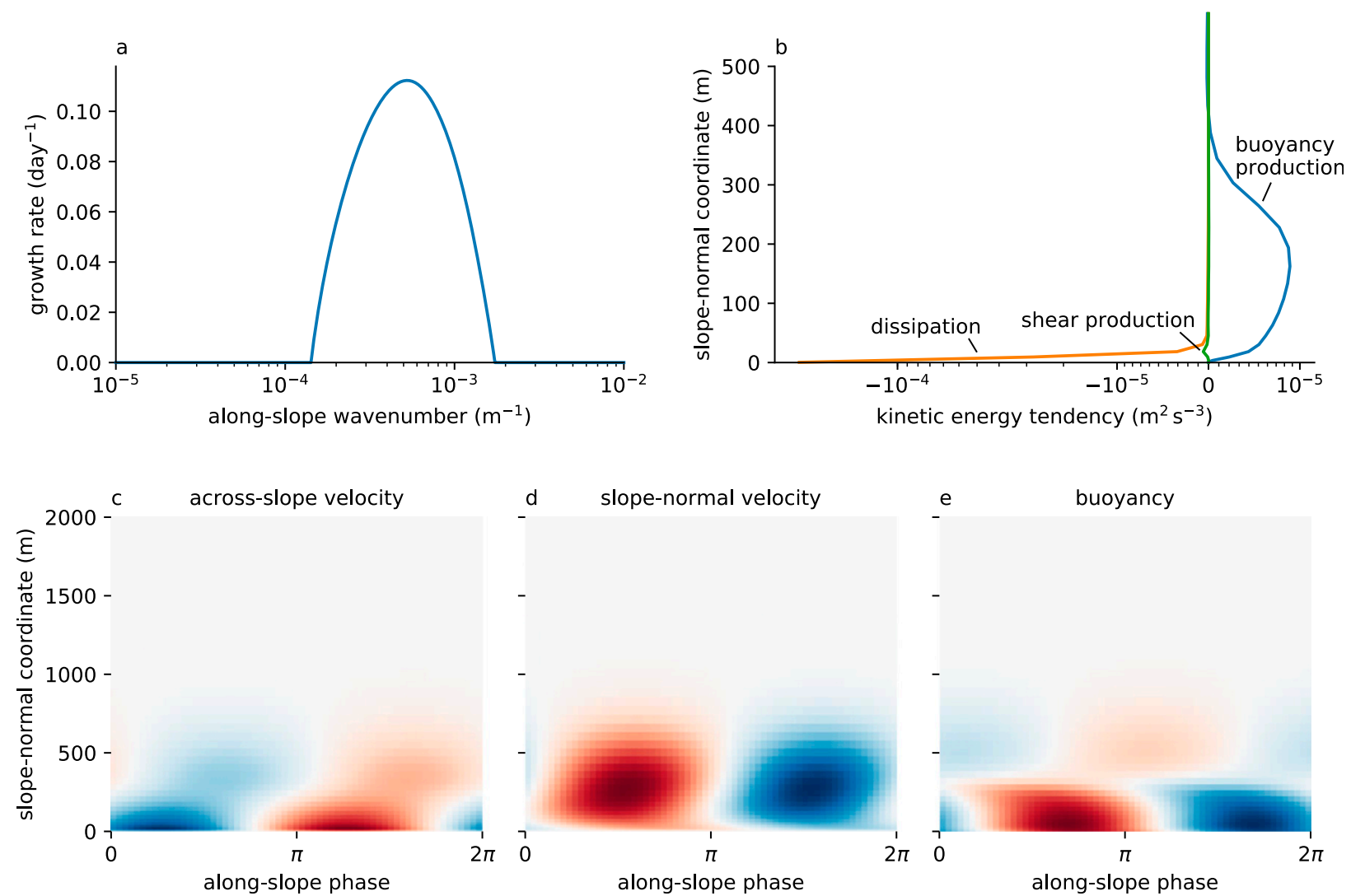

FIG. 5. Linear stability analysis of the steady one-dimensional solution: (a) growth rate as a function of along-slope wavenumber (peak growth occurs at a wavelength of about $12 \mathrm{~km}$ ); (b) energy budget of the most unstable mode, showing it grows by baroclinic production and is significantly damped by vertical diffusion in the bottom Ekman layer (note the arsinh scale of the abscissa); (c)-(e) structure of the most unstable mode in the along-slope-slope normal plane (positive values are red; negative values are blue).

The eddy kinetic energy is

$$
K=\frac{1}{2}\left\langle\overline{u^{12}}+\overline{v^{12}}\right\rangle
$$

The overline denotes an average in the plane of the slope, $A^{\prime}=A-\bar{A}$ denotes perturbations in the plane of the slope, and the angle brackets denote a slope-normal integral over the domain. Eddy kinetic energy can be created by buoyancy production (first term on the right) and shear production (second term on the right), and it is lost to dissipation (third term on the right) and to hyperviscosity (fourth term on the right). The buoyancy production has two terms because the coordinate system is rotated with respect to gravity.

As expected from the linear stability calculation (Fig. 5b), eddy kinetic energy is created by a slight dominance of buoyancy production in the outer mixing layer over dissipation in the bottom Ekman layer (Figs. 7a,b). Both buoyancy production and dissipation keep increasing in the nonlinear phase of the evolution, but they level out and slightly decrease after $t=150$ days.
The eddy kinetic energy itself also levels out and starts decreasing shortly after that. Neither shear production nor hyperviscosity plays a significant role in the energetics.

Once the baroclinic perturbations have reached significant amplitude around $t=75$ days, they start modifying the mean state (Figs. 7c,d). The sliding of dense water under light water that energizes the instability also restratifies the mean state (Figs. $3 c$ and $7 c$ ). Once the perturbations have reached significant amplitude, it takes just 50 days for the eddies to substantially enhance the stratification in the mixing layer. The restratification is largest just above the bottom Ekman layer, but the entire outer layer restratifies, reaching stratification values of the same order as in the far field around $t=150$ days.

The restratification is attended by a reduction in the along-slope shear (Fig. 2d, green curve; Fig. 7d). This is to be expected because throughout the evolution of the flow the along-slope shear remains in approximate thermal wind balance $f \bar{v}_{z} \cos \theta=-\bar{b}_{z} \sin \theta$, where the 

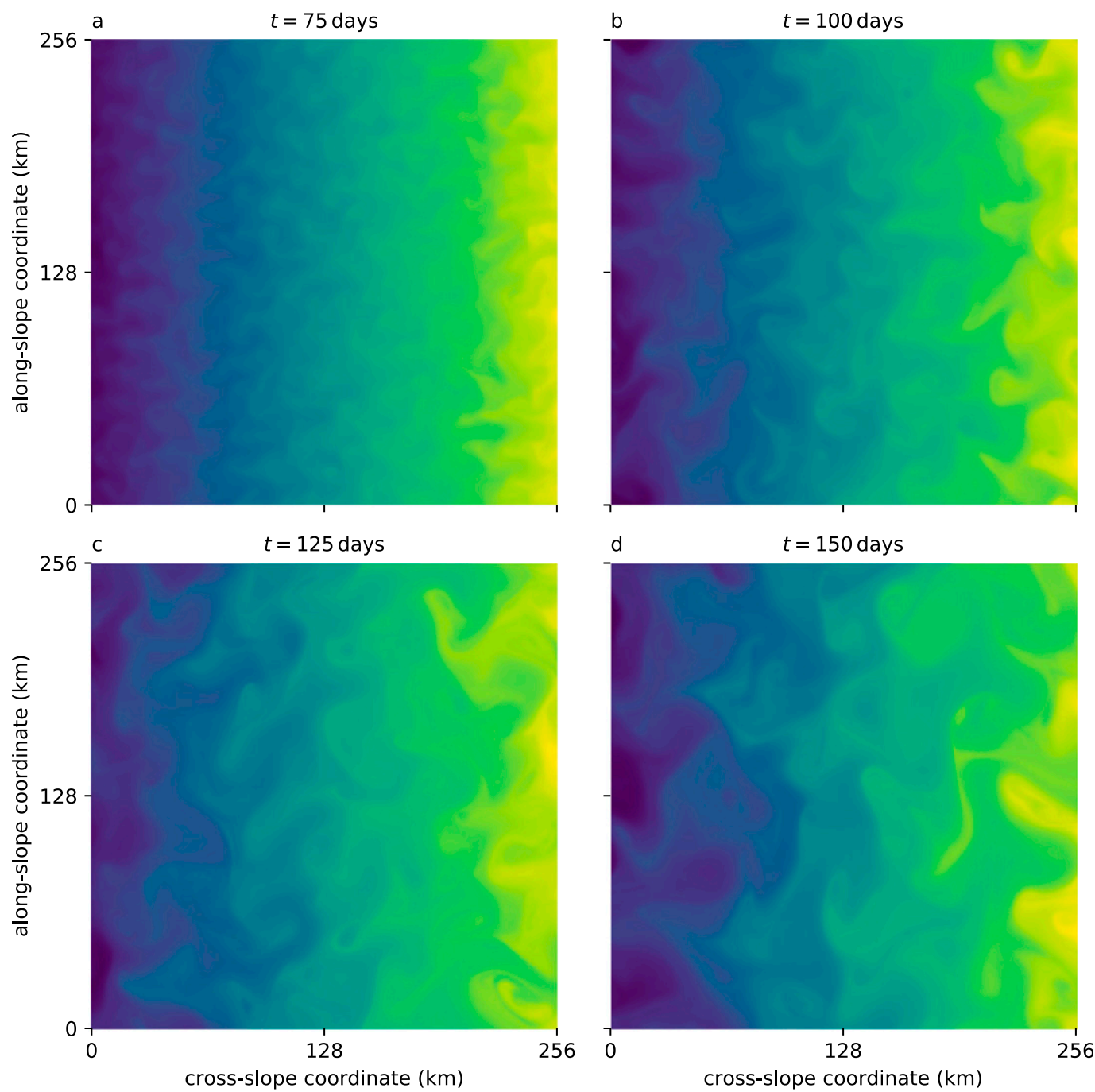

FIG. 6. Snapshots of the buoyancy field $N^{2}(z \cos \theta+x \sin \theta)+b$ from the fully nonlinear three-dimensional simulation started from the one-dimensional steady state (experiment 1 ), showing the development and subsequent evolution of baroclinic eddies. The snapshots are taken at a slope-normal distance $z=100 \mathrm{~m}$ and at the times denoted above each panel. The densest water is shown in purple and the most buoyant water in yellow.

right side is the mean buoyancy gradient in the true horizontal. Initially, the perturbation $\bar{b}_{z}$ is strongly negative and opposes the background contribution $N^{2} \cos \theta$, so $\bar{v}_{z}$ is strongly negative as well $(f<0)$. As the mixing layer restratifies, $\bar{b}_{z}$ and thus $\bar{v}_{z}$ become less negative. The reduction in shear is induced by the familiar vertical momentum flux effected by baroclinic eddies (e.g., Rhines and Young 1982).

\section{b. Maintenance of stratification by baroclinic eddies}

To show that baroclinic eddies emerge and enter the leading-order balances also in an abyssal mixing layer that is more realistically stratified, I consider a second experiment that is initialized with the observed stratification. To obtain smooth initial conditions, I fit the functional form of the outer-layer stratification profile of the one-dimensional solution - that is, the first two terms on the right side of (10) to the observed stratification, considering $S \sigma /(1+S \sigma)$ a free parameter. The best fit is obtained with $S \sigma /(1+S \sigma)=0.35$ (Fig. 2a, black curve; Fig. 3a). I assume the along-slope flow to be in thermal wind balance and to vanish at the bottom (Fig. 2d, black curve), and I set the cross-slope flow to zero everywhere. Small random noise is again added to the initial buoyancy field, and the setup, discretization, and parameters are the same as in experiment 1 above, except that now a larger time step of $1 \mathrm{~h}$ is used.

To isolate the effect of baroclinic eddies, I compare this three-dimensional experiment 2 to the onedimensional evolution obtained from (2)-(4), with the tendency terms reinstated: 

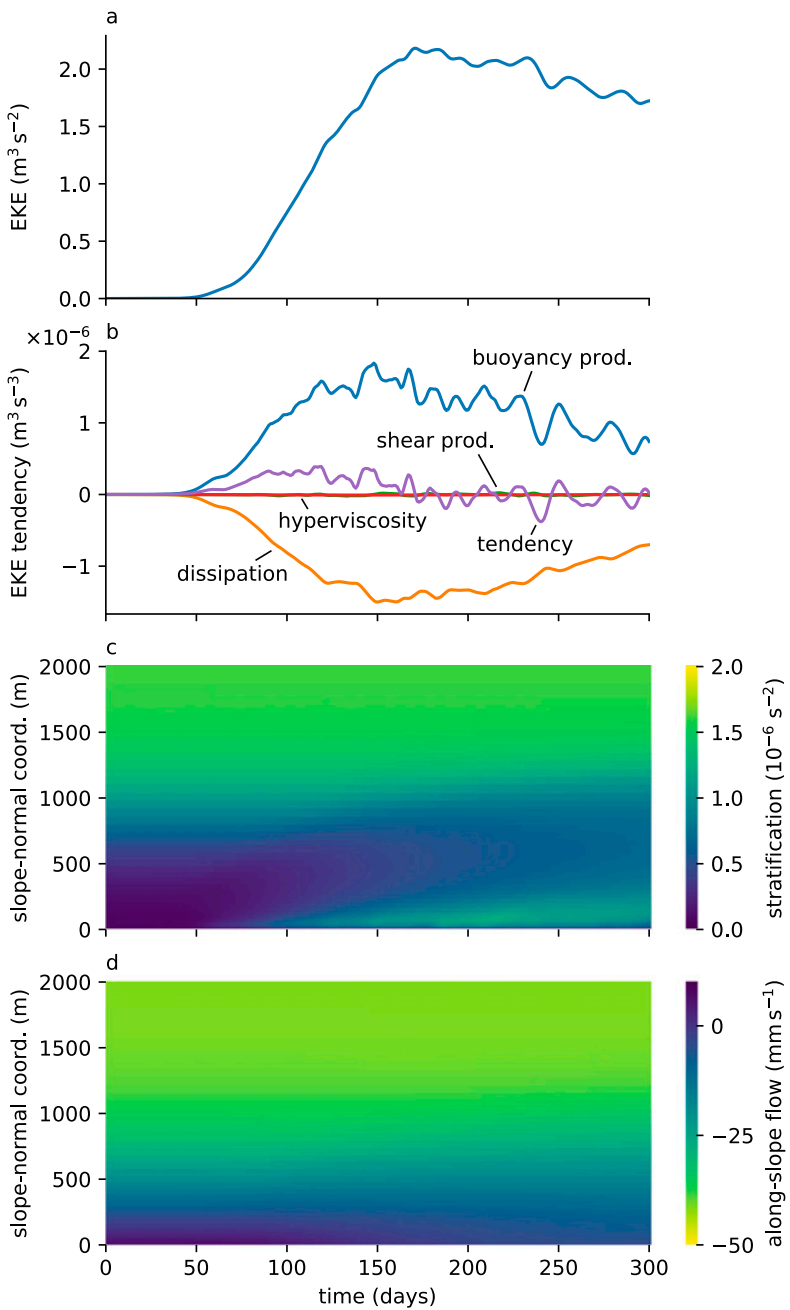

FIG. 7. Evolution of the three-dimensional solution initialized with the one-dimensional steady state (experiment 1 ). (a) The time series of eddy kinetic energy (vertically integrated) shows that finite-amplitude baroclinic eddies emerge around $t=50$ days.

(b) The terms in the eddy kinetic energy budget reveal that a positive kinetic energy tendency is generated in the growth phase by a slight dominance of buoyancy production over dissipation; shear production and hyperviscosity play negligible roles. (c) The initially weak stratification in the mixing layer is increased by the action of baroclinic eddies once they have reached significant amplitude around $t=100$ days. (d) The along-slope flow remains in approximate thermal wind balance, which means that a reduction in shear attends the restratification.

$$
\begin{aligned}
u_{t}-f v \cos \theta & =b \sin \theta+\left(\nu u_{z}\right)_{z}, \\
v_{t}+f u \cos \theta & =\left(\nu v_{z}\right)_{z}, \\
b_{t}+u N^{2} \sin \theta & =\left[\kappa\left(N^{2} \cos \theta+b_{z}\right)\right]_{z} .
\end{aligned}
$$

In these one-dimensional dynamics, the stratification in the mixing layer quickly erodes (Fig. 8). After 6000 days, the bottom $500 \mathrm{~m}$ have become nearly unstratified. While 6000 days is too short for this solution to reach the steady state of the previous section, which requires diffusion into the far field (cf. MacCready and Rhines 1991; Garrett et al. 1993), it is long enough for the vigorous near-bottom mixing to effectively homogenize the fluid in the bottom few hundred meters. The diffusive time scale based on the bottom diffusivity and a vertical distance of $500 \mathrm{~m}$ is on the order of a year (cf. Thompson and Johnson 1996).

The evolution is markedly different in the threedimensional case (Fig. 9). The erosion of stratification lasts only 500 days, after which baroclinic eddies have reached large-enough amplitude to restratify the mixing layer (Fig. 9c). Like in experiment 1, the eddies are energized by a slight imbalance between buoyancy production and dissipation in the Ekman layer (Figs. 9a,b). Both the eddy kinetic energy and the terms in the energy budget are an order of magnitude smaller than in the experiment started from the one-dimensional steady state, but the baroclinic restratification is still strong enough to maintain the mixing-layer stratification close to the observed value (Fig. 9c). The average stratification over the time interval 3000-6000 days is in remarkable agreement with the observations (Fig. 2a, red curve; Figs. 3a,d).

It is interesting to note that the stratification observed in Brazil Basin mixing layers corresponds to isopycnal slopes that are of the same order as the large-scale topographic slope (Figs. 1b and 3a). This may not be a coincidence. Baroclinic eddies cause strong restratification of abyssal mixing layers, but the instability that gives rise to the eddies can be stabilized by the topographic slope if the topographic and isopycnal slopes are comparable (e.g., Blumsack and Gierasch 1972; Wenegrat et al. 2018). The observed hydrography may thus be the result of baroclinic adjustment to a marginally stable state. More simulations and analysis of observations, however, are needed to test this conjecture.

\section{Implications for the abyssal overturning circulation}

The presence of strong stratification in abyssal mixing layers is crucial for bottom-enhanced turbulence to achieve water mass transformation and thus to affect the overturning circulation. It is the stratification that allows the turbulence to generate large diabatic buoyancy fluxes, whose convergence and divergence causes the dipole of up- and downwelling that has emerged as a key feature of the diabatic circulation of the abyssal ocean (de Lavergne et al. 2016; Ferrari et al. 2016; McDougall and Ferrari 2017; de Lavergne et al. 2017; Holmes et al. 2018; Callies and Ferrari 2018b). The magnitude of this dipole is substantial: an estimate based on the observed stratification and a semiempirical mixing map yields 


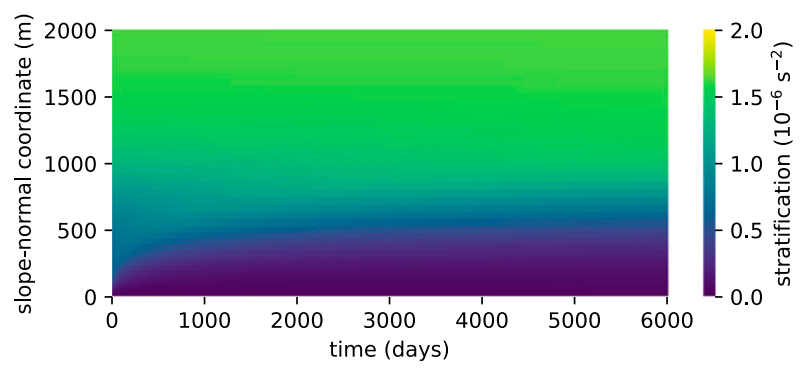

FIG. 8. Evolution of the one-dimensional solution initialized with the observed stratification. The vigorous near-bottom mixing quickly erodes the stratification in the bottom few hundred meters if unopposed by baroclinic eddies.

$40 \mathrm{~Sv}$ of upwelling and $30 \mathrm{~Sv}$ of downwelling across the isopycnal bounding Antarctic Bottom Water (Ferrari et al. 2016).

A similar estimate for the dipole strength can be made by extrapolating the Brazil Basin observations: assuming a planar slope, the diabatic transports can be inferred by taking the differences of $\psi N^{2} \sin \theta=-F^{z}=\Gamma \varepsilon$ across the up- and downwelling layers (cf. Garrett 1991; McDougall and Ferrari 2017). The difference between the far-field dissipation rate and the bottommost observed value gives an estimate for the downwelling over the observed layer; the bottommost observed value itself gives an estimate for the upwelling below. Multiplying the resulting numbers by an along-slope distance of $100000 \mathrm{~km}$, about the length of the global oceanic ridge system, yields $16 \mathrm{~Sv}$ of downwelling and $18 \mathrm{~Sv}$ of upwelling. These numbers are not intended to be serious global estimates, which would have to take variations in dissipation rate, stratification, and slope into account; the multiplication by an alongslope length simply allows me to reason about more familiar numbers. The fact that the result has the same order of magnitude as Ferrari et al.'s estimate, however, suggests that the diabatic transports of the Brazil Basin may not be atypical.

Such a strong dipole in the diabatic transport would be impossible if the dynamics in abyssal mixing layers were one-dimensional. The analytical solution (7) shows that the downwelling transport in the outer layer would be $\kappa_{1} \cot \theta S \sigma /(1+S \sigma)$. Using the Brazil Basin parameters and again multiplying by an along-slope length of $100000 \mathrm{~km}$ gives $0.2 \mathrm{~Sv}$. The upwelling transport in the inner layer would be $\kappa_{1} \cot \theta S \sigma /(1+S \sigma)+\kappa_{0} \cot \theta$, giving $3 \mathrm{~Sv}$ for Brazil Basin parameters. The dipole strength of $0.2 \mathrm{~Sv}$ is drastically weaker than the $16 \mathrm{~Sv}$ inferred from the observed dissipation rates. This is a direct consequence of the insufficient restratification by mean flows in one-dimensional dynamics: if there is only weak stratification that mixing can act on, the resulting diabatic
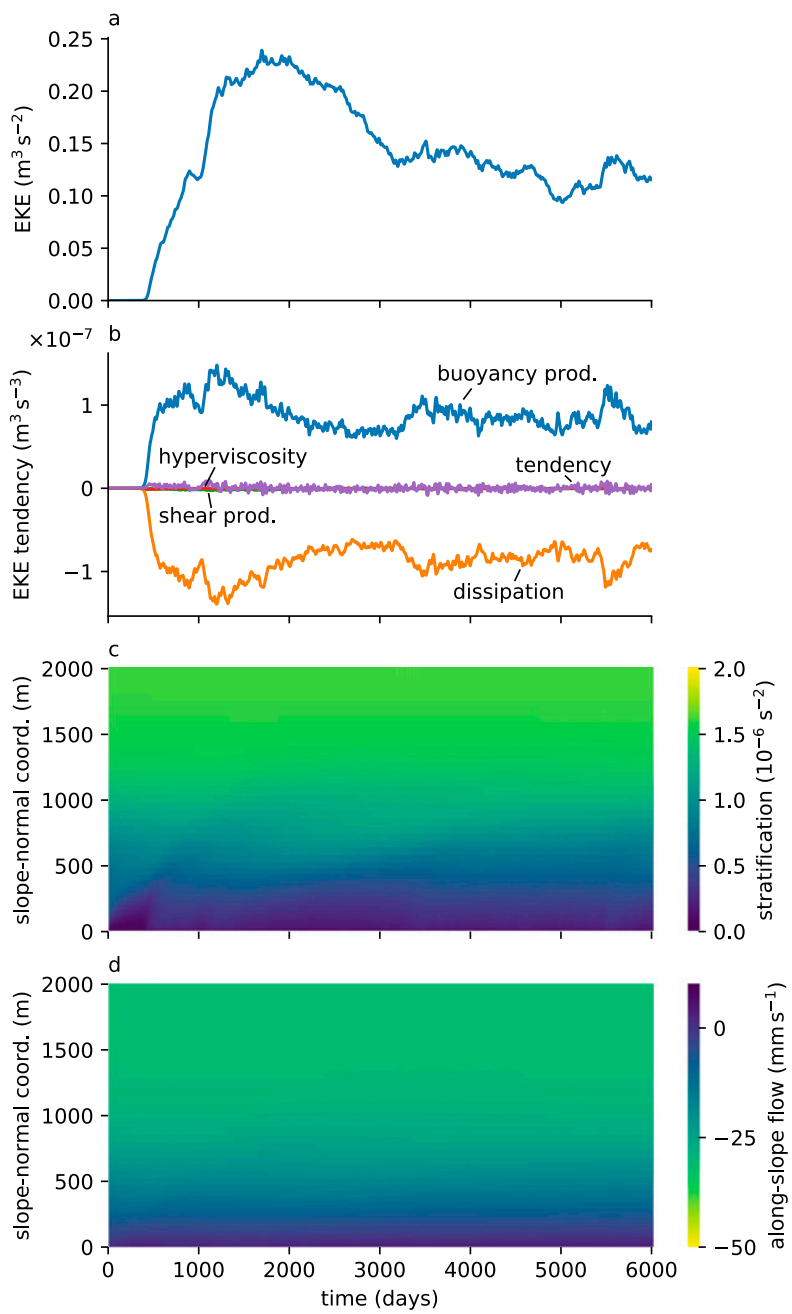

FIG. 9. Evolution of the three-dimensional solution initialized with the observed stratification (experiment 2). (a) The time series of eddy kinetic energy (vertically integrated) show that finite-amplitude baroclinic eddies emerge just after $t=500$ days. (b) The terms in the eddy kinetic energy budget show that kinetic energy is generated again by a slight dominance of buoyancy production over dissipation; shear production and hyperviscosity are small. (c) The stratification starts eroding initially, but once baroclinic eddies have emerged, the stratification is restored to and maintained near the realistic initial value. (d) The along-slope flow shows little change over the course of the simulation, remaining in approximate thermal wind balance.

buoyancy fluxes are reduced in magnitude, and the upand downwelling dipole is weak.

In the three-dimensional dynamics, on the other hand, baroclinic restratification restores the estimate of the up- and downwelling dipole to what is inferred from observations. By laterally sliding dense water under light water, baroclinic eddies allow small-scale turbulence to act on stratified water. Given the same turbulent diffusivity, the enhanced stratification produces diabatic fluxes and thus water mass transformation that is much 
larger than in the one-dimensional case. This suggests that an up- and downwelling dipole of a few tens of Sverdrups can be achieved if submesoscale baroclinic eddies counter the homogenizing effect of bottomintensified mixing in abyssal mixing layers.

This result raises questions about the representation of the abyssal overturning circulation in global ocean models. These models have coarse resolution in both the horizontal and vertical (especially in the abyssal ocean) and thus heavily distort the dynamics of abyssal mixing layers. The above discussion suggests furthermore that using a realistically bottom-intensified mixing profile and improving the vertical resolution may not be sufficient to capture the strong up- and downwelling dipole. One may speculate that if topography (which is necessarily smoothed), far-field stratification, and mixing coefficients vary only slowly in the horizontal (in a WKB sense), the dynamics will locally be similar to the onedimensional dynamics discussed above (cf. Callies and Ferrari 2018b). Global models with high vertical but low horizontal resolution would thus suffer from the same problems as the one-dimensional solution. The stratification in abyssal mixing layers would be eroded by the bottom-intensified mixing, restratification by crossslope mean flows would be weak, and no substantial upand downwelling dipole would emerge. If baroclinic restratification is the dominant mechanism that maintains stratification, one will have to resolve or parameterize submesoscale baroclinic eddies in abyssal mixing layers in order to produce a realistic abyssal overturning circulation.

\section{Discussion}

The weak mean-flow restratification produced by one-dimensional dynamics and the strong baroclinic restratification produced by three-dimensional dynamics suggest a crucial role of submesoscale baroclinic eddies in the maintenance of mixing-layer stratification. It should be kept in mind, however, that I made a number of assumptions in the simplified cases considered above.

First, the turbulent momentum and buoyancy fluxes produced by internal wave breaking were represented with simple diffusive closures, and the turbulent Prandtl number was set to unity. It is neither clear that momentum fluxes generated by internal wave breaking are generally downgradient, nor that the turbulent viscosity should equal the turbulent diffusivity. The onedimensional solution can be brought into much closer agreement with the observations if the Prandtl number

\footnotetext{
${ }^{6}$ This fit was obtained using numerical solutions because the assumption $q h \gg 1$ breaks down for large Prandtl numbers.
}

is increased to order 100 - an optimal $\mathrm{fit}^{6}$ is obtained with $\sigma=230$-but it is unclear whether internal wave breaking can achieve such large momentum fluxes. It seems more likely that the momentum fluxes are enhanced by baroclinic eddies and that an increased Prandtl number amounts to a parameterization of these eddies (e.g., Rhines and Young 1982; Young 2012). But more work is needed to clarify this issue, for example, by explicitly representing the waves and their breaking (cf. Nikurashin and Legg 2011).

The diffusive dynamics also do not capture any logarithmic turbulent layers adjacent to the bottom (e.g., Townsend 1976). Since the focus here is on the turbulence generated aloft by internal wave breaking, this should not affect the conclusions. It should be kept in mind, however, that the diffusive parameterization glosses over our ignorance about the turbulence in the bottom few tens of meters.

Another consequence of the diffusive parameterization is that feedbacks between the subinertial dynamics considered here and the waves and turbulence that produce the mixing are excluded. In reality, the wave generation and breaking would itself be strongly modulated by the stratification in the mixing layer. The above cases with fixed mixing coefficients are thus best thought of as consistency tests: Are the subinertial dynamics induced by the turbulence capable of maintaining the observed mean state that the turbulence generation depends on? That this test is passed only if submesoscale eddies are present is strongly suggestive of their importance, but the full dynamics with interactive waves, turbulence, and baroclinic eddies should be considered in future work.

Second, the solutions considered above take into account the large-scale topographic slope only. The smallscale topography - that is, the abyssal hills and fracture zones that are responsible for the bottom-enhanced turbulence in the first place-entered the calculations only indirectly through the bottom-enhanced mixing coefficients. These topographic features may steer mean flows and thus change their ability to maintain stratification. For example, the walls of fracture zone canyons may block flows along the large-scale slope of the Mid-Atlantic Ridge (e.g., Thurnherr and Speer 2003). A rough estimate of this blocking effect can be obtained by setting $v=0$ or equivalently $f=0$; that is, by reverting to nonrotating dynamics (Dell 2013). This would amount to $S \sigma /(1+S \sigma) \rightarrow 1$ and thus yield a dramatic increase in stratification and water mass transformation (cf. Clément and Thurnherr 2018). It remains unclear, however, how strong a stratification can be maintained in the presence of realistically bottomintensified mixing both within and outside of canyons and over more general irregular topography. The 
topography may also modulate baroclinic instability (e.g., de Szoeke 1983) and the resulting baroclinic eddies, effects that should also be addressed by future work.

Third, the setup in a sloping coordinate system with a prescribed far-field stratification restricts attention to the local dynamics. Nonlocal effects, such as changes in the large-scale topographic slope, varying far-field stratification, or the change of the inertial frequency with latitude, are all neglected. Such variations can cause convergences and divergences of the cross-slope mean flow and thus give rise to exchange with the far field (e.g., Phillips et al. 1986; McDougall 1989; Kunze et al. 2012; Dell and Pratt 2015; Holmes et al. 2018). Leading-order changes to the mean-flow restratification, however, are not expected: if the variations are slow, a WKB approach suggests that the leading-order dynamics are still one-dimensional locally, and mean-flow restratification should remain weak.

Beyond exploring the above questions in more realistic numerical simulations, one should look for evidence of submesoscale baroclinic eddies in observations. Moored current meters deployed in a Brazil Basin fracture zone canyon do show substantial subinertial variability that is bottom intensified (Thurnherr et al. 2005; Toole 2007; Clément et al. 2017), as expected for baroclinic eddies generated in mixing layers. But a careful analysis and possibly more observations are needed to discriminate against other sources of subinertial variability.

If the local dynamics on a planar large-scale slope capture the essence of abyssal mixing layers, effective baroclinic restratification should generally appear in abyssal mixing layers. The problem is determined completely by the following set of nondimensional parameters: the slope Burger number $S$, the Ekman number $E=\nu_{1} / f h^{2}$ based on the decay scale of mixing $h$, the bottom-enhancement ratio $\kappa_{1} / \kappa_{0}$, and the turbulent Prandtl number $\sigma$. In the abyssal ocean, where stratification is relatively weak and large-scale topographic slopes are gentle, $S \ll 1$ is typical (except very close to the equator). When turbulence is produced by the breaking of topographically generated internal waves, it typically extends far beyond the Ekman layer $\left(|E|^{1 / 2} \ll 1\right)$ and is much stronger than in the far field $\left(\kappa_{1} \gg \kappa_{0}\right.$; e.g., Waterhouse et al. 2014). The calculations presented above are in this typical regime: $S=2.2 \times 10^{-3}$, $|E|=6.5 \times 10^{-4}, \kappa_{1} / \kappa_{0}=35$, and $\sigma=1$. For baroclinic instabilities to exist, it is crucial that the outer layer predicted by one-dimensional dynamics is much thicker than the inner layer, which requires $\log \left(\kappa_{1} / \kappa_{0}\right) \gg|E|^{1 / 2}$ $(1+S \sigma)$, and that the outer-layer stratification predicted by one-dimensional theory is much weaker than in the far field, which is the case if $S \sigma \ll 1$ and $\kappa_{1} / \kappa_{0} \gg 1$. These conditions are typically met where there is strong topographically induced mixing, so baroclinic restratification should be widespread in abyssal mixing layers.

\section{Conclusions}

The results of this paper raise the possibility that submesoscale baroclinic eddies are instrumental in maintaining stratification in abyssal mixing layers against the homogenizing tendency of bottom-intensified mixing. The restratification of abyssal mixing layers is crucial because it allows the topographically induced turbulence to continually achieve water mass transformation and produce a diapycnal up- and downwelling dipole of a few tens of Sverdrups globally. Baroclinic restratification may thus help shape the overturning circulation of the abyssal ocean.

Considering the local dynamics of abyssal mixing layers on slopes, this paper revealed a stark difference between one- and three-dimensional dynamics. If the flow is onedimensional, that is, invariant in the plane of the slope, the homogenizing tendency of mixing is countered by cross-slope mean flows only. With parameters appropriate for the Brazil Basin, one-dimensional solutions produce mean flows that are much too weak to maintain the observed stratification. But the weakly stratified mixing layers produced by such one-dimensional dynamics are unstable to baroclinic perturbations. In three-dimensional dynamics, submesoscale baroclinic eddies emerge and restratify mixing layers by laterally sliding dense water under light water. These eddies can effectively maintain the observed stratification.

While baroclinic restratification is largely adiabatic and does not cause water mass transformation directly, it does modify the stratification that is mixed up by small-scale turbulence. Given the same mixing coefficients, the enhanced stratification allows for much stronger diabatic fluxes and thus water mass transformation. The up- and downwelling dipole thought to be generated by bottomintensified mixing would be much weaker if the mixinglayer dynamics were one-dimensional and mixing layers were largely unstratified. Baroclinic restratification in abyssal mixing layers could therefore directly affect the large-scale overturning circulation of the abyssal ocean.

Global models typically do not resolve these submesoscale instabilities in the abyss because of a lack of both horizontal and vertical resolution. If the abyssal overturning circulation is indeed affected by submesoscale baroclinic restratification, incorporating its effects into global models is imperative. This could be done either by drastically increasing the resolution or by devising a parameterization similar to that of submesoscale baroclinic restratification in the surface mixed layer (Fox-Kemper et al. 2011). 
Acknowledgments. I would like to thank the developers of Dedalus (dedalus-project.org), which was used for all computations presented in this paper. In particular, I would like to thank Keaton Burns, who also assisted with the setup and optimization of the computations. Chris Garrett and Carl Wunsch gave valuable feedback on the manuscript, and anonymous reviewers provided useful suggestions.

\section{REFERENCES}

Armi, L., 1978: Some evidence for boundary mixing in the deep ocean. J. Geophys. Res., 83, 1971-1979, https://doi.org/10.1029/ JC083iC04p01971.

— 1979: Reply to comments by C. Garrett. J. Geophys. Res., 84, 5097-5098, https://doi.org/10.1029/JC084iC08p05097.

Bender, C. M., and S. A. Orszag, 1999: Advanced Mathematical Methods for Scientists and Engineers I. Springer-Verlag, 593 pp., https://doi.org/10.1007/978-1-4757-3069-2.

Blumsack, S. L., and P. J. Gierasch, 1972: Mars: The effects of topography on baroclinic instability. J. Atmos. Sci., 29, 1081-1089, https://doi.org/10.1175/1520-0469(1972)029<1081: MTEOTO $>2.0 . \mathrm{CO} ; 2$.

Boccaletti, G., R. Ferrari, and B. Fox-Kemper, 2007: Mixed layer instabilities and restratification. J. Phys. Oceanogr., 37, 2228 2250, https://doi.org/10.1175/JPO3101.1.

Bretherton, F. P., and M. Karweit, 1975: Mid-ocean mesoscale modeling. Numerical Models of Ocean Circulation, R. O. Reid, A. R. Robinson, and K. Bryan, Eds., National Academy of Sciences, 237-249.

Burns, K. J., G. M. Vasil, J. S. Oishi, D. Lecoanet, and B. Brown, 2016: ASCL code record. Astrophysics Source Code Library, ascl:1603.015, http://ascl.net/1603.015.

Callies, J., and R. Ferrari, 2018a: Baroclinic instability in the presence of convection. J. Phys. Oceanogr., 48, 45-60, https:// doi.org/10.1175/JPO-D-17-0028.1.

— , and _ 2018b: Dynamics of an abyssal circulation driven by bottom-intensified mixing on slopes. J. Phys. Oceanogr., 48 , 1257-1282, https://doi.org/10.1175/JPO-D-17-0125.1.

— , G. Flierl, R. Ferrari, and B. Fox-Kemper, 2016: The role of mixed-layer instabilities in submesoscale turbulence. J. Fluid Mech., 788, 5-41, https://doi.org/10.1017/jfm.2015.700.

Clément, L., and A. M. Thurnherr, 2018: Abyssal upwelling in midocean ridge fracture zones. Geophys. Res. Lett., 45, 2424-2432, https://doi.org/10.1002/2017GL075872.

,$- \ldots$, and L. C. St. Laurent, 2017: Turbulent mixing in a deep fracture zone on the Mid-Atlantic Ridge. J. Phys. Oceanogr., 47, 1873-1896, https://doi.org/10.1175/JPO-D-16-0264.1.

de Lavergne, C., G. Madec, J. Le Sommer, A. J. G. Nurser, and A. C. Naveira Garabato, 2016: On the consumption of Antarctic Bottom Water in the abyssal ocean. J. Phys. Oceanogr., 46, 635-661, https://doi.org/10.1175/JPO-D-14-0201.1.

,,-- F. Roquet, R. M. Holmes, and T. J. McDougall, 2017: Abyssal ocean overturning shaped by seafloor distribution. Nature, 551, 181-186, https://doi.org/10.1038/nature24472.

Dell, R. W., 2013: Boundary layer dynamics and deep ocean mixing in Mid-Atlantic Ridge canyons. Ph.D. thesis, MIT-WHOI Joint Program in Oceanography, 163 pp., https://doi.org/ 10.1575/1912/5740.
— and L. J. Pratt, 2015: Diffusive boundary layers over varying topography. J. Fluid Mech., 769, 635-653, https://doi.org/ 10.1017/jfm.2015.88.

de Szoeke, R. A., 1983: Baroclinic instability over wavy topography. J. Fluid Mech., 130, 279-298, https://doi.org/10.1017/ S0022112083001093.

Efron, B., and T. Hastie, 2016: Computer Age Statistical Inference. Cambridge University Press, 475 pp.

Ferrari, R., A. Mashayek, T. J. McDougall, M. Nikurashin, and J.-M. Campin, 2016: Turning ocean mixing upside down. J. Phys. Oceanogr., 46, 2239-2261, https://doi.org/10.1175/JPO-D-15-0244.1.

Fox-Kemper, B., R. Ferrari, and R. W. Hallberg, 2008: Parameterization of mixed layer eddies. Part I: Theory and diagnosis. J. Phys. Oceanogr., 38, 1145-1165, https://doi.org/ 10.1175/2007JPO3792.1.

— eddies. III: Implementation and impact in global ocean climate simulations. Ocean Modell., 39, 61-78, https://doi.org/ 10.1016/j.ocemod.2010.09.002.

Garrett, C., 1979: Comment on 'Some evidence for boundary mixing in the deep ocean' by Laurence Armi. J. Geophys. Res., 84, 5095, https://doi.org/10.1029/JC084iC08p05095.

, 1990: The role of secondary circulation in boundary mixing. J. Geophys. Res., 95, 3181-3188, https://doi.org/10.1029/ JC095iC03p03181.

_- 1991: Marginal mixing theories. Atmos.-Ocean, 29, 313-339, https://doi.org/10.1080/07055900.1991.9649407.

, and E. Kunze, 2007: Internal tide generation in the deep ocean. Annu. Rev. Fluid Mech., 39, 57-87, https://doi.org/ 10.1146/annurev.fluid.39.050905.110227.

_ , P. MacCready, and P. B. Rhines, 1993: Boundary mixing and arrested Ekman layers: Rotating stratified flow near a sloping boundary. Annu. Rev. Fluid Mech., 25, 291-323, https://doi.org/ 10.1146/annurev.fl.25.010193.001451.

Gregg, M. C., E. A. D'Asaro, J. J. Riley, and E. Kunze, 2018: Mixing efficiency in the ocean. Annu. Rev. Mar. Sci., 10, 443473, https://doi.org/10.1146/annurev-marine-121916-063643.

Haidvogel, D. B., and I. M. Held, 1980: Homogeneous quasigeostrophic turbulence driven by a uniform temperature gradient. J. Atmos. Sci., 37, 2644-2660, https://doi.org/10.1175/ 1520-0469(1980)037<2644:HQGTDB > 2.0.CO;2.

Holmes, R. M., C. de Lavergne, and T. J. McDougall, 2018: Ridges, seamounts, troughs, and bowls: Topographic control of the dianeutral circulation in the abyssal ocean. J. Phys. Oceanogr., 48, 861-882, https://doi.org/10.1175/JPO-D-17-0141.1.

Jayne, S. R., 2009: The impact of abyssal mixing parameterizations in an ocean general circulation model. J. Phys. Oceanogr., 39, 1756-1775, https://doi.org/10.1175/2009JPO4085.1.

Kunze, E., C. MacKay, E. E. McPhee-Shaw, K. Morrice, J. B. Girton, and S. R. Terker, 2012: Turbulent mixing and exchange with interior waters on sloping boundaries. J. Phys. Oceanogr., 42, 910927, https://doi.org/10.1175/JPO-D-11-075.1.

Ledwell, J. R., E. T. Montgomery, K. L. Polzin, L. C. St. Laurent, R. W. Schmitt, and J. M. Toole, 2000: Evidence for enhanced mixing over rough topography in the abyssal ocean. Nature, 403, 179-182, https://doi.org/10.1038/35003164.

Legg, S., and K. M. H. Huijts, 2006: Preliminary simulations of internal waves and mixing generated by finite amplitude tidal flow over isolated topography. Deep-Sea Res. II, 53, 140-156, https://doi.org/10.1016/j.dsr2.2005.09.014.

Lumpkin, R., and K. Speer, 2007: Global ocean meridional overturning. J. Phys. Oceanogr., 37, 2550-2562, https://doi.org/ 10.1175/JPO3130.1. 
MacCready, P., and P. B. Rhines, 1991: Buoyant inhibition of Ekman transport on a slope and its effect on stratified spin-up. J. Fluid Mech., 223, 631-661, https://doi.org/10.1017/S0022112091001581.

McDougall, T. J., 1989: Dianeutral advection. Parameterization of SmallScale Processes: Proc. 'Aha Huliko'a Hawaiian Winter Workshop, Honolulu, HI, University of Hawai'i at Mānoa, 289-315.

— the Gibbs Seawater (GSW) Oceanographic Toolbox. 3rd ed. SCOR/IAPSO WG127 Rep., 34 pp.

_- and R. Ferrari, 2017: Abyssal upwelling and downwelling driven by near-boundary mixing. J. Phys. Oceanogr., 47, 261283, https://doi.org/10.1175/JPO-D-16-0082.1.

Muller, C. J., and O. Bühler, 2009: Saturation of the internal tides and induced mixing in the abyssal ocean. J. Phys. Oceanogr., 39, 2077-2096, https://doi.org/10.1175/2009JPO4141.1.

Munk, W. H., 1966: Abyssal recipes. Deep-Sea Res. Oceanogr. Abstr., 13, 707-730, https://doi.org/10.1016/0011-7471(66)90602-4.

Nikurashin, M., and R. Ferrari, 2010: Radiation and dissipation of internal waves generated by geostrophic motions impinging on small-scale topography: Theory. J. Phys. Oceanogr., 40, 1055-1074, https://doi.org/10.1175/2009JPO4199.1.

_ ternal tides generated at rough topography. J. Phys. Oceanogr., 41, 378-395, https://doi.org/10.1175/2010JPO4522.1.

Osborn, T. R., 1980: Estimates of the local rate of vertical diffusion from dissipation measurements. J. Phys. Oceanogr., 10, 83-89, https:// doi.org/10.1175/1520-0485(1980)010<0083:EOTLRO > 2.0.CO;2.

Pedlosky, J., 1987: Geophysical Fluid Dynamics. 2nd ed. Springer, $710 \mathrm{pp}$.

Phillips, O. M., 1970: On flows induced by diffusion in a stably stratified fluid. Deep-Sea Res. Oceanogr. Abstr., 17, 435-440, https://doi.org/10.1016/0011-7471(70)90058-6.

__, J.-H. Shyu, and H. Salmun, 1986: An experiment on boundary mixing: Mean circulation and transport rates. J. Fluid Mech., 173, 473-499, https://doi.org/10.1017/ S0022112086001234.

Polzin, K. L., J. M. Toole, J. R. Ledwell, and R. W. Schmitt, 1997: Spatial variability of turbulent mixing in the abyssal ocean. Science, 276, 93-96, https://doi.org/10.1126/science.276.5309.93.

Rhines, P. B., and W. R. Young, 1982: Homogenization of potential vorticity in planetary gyres. J. Fluid Mech., 122, 347-367, https://doi.org/10.1017/S0022112082002250.

Salmon, R., 1980: Baroclinic instability and geostrophic turbulence. Geophys. Astrophys. Fluid Dyn., 15, 167-211, https:// doi.org/10.1080/03091928008241178.

Smith, W. H. F., and D. T. Sandwell, 1997: Global sea floor topography from satellite altimetry and ship depth soundings. Science, 277, 1956-1962, https://doi.org/10.1126/science.277.5334.1956.
St. Laurent, L. C., J. M. Toole, and R. W. Schmitt, 2001: Buoyancy forcing by turbulence above rough topography in the abyssal Brazil Basin. J. Phys. Oceanogr., 31, 3476-3495, https://doi.org/ 10.1175/1520-0485(2001)031<3476:BFBTAR > 2.0.CO;2.

—, A. C. Naveira Garabato, J. R. Ledwell, A. M. Thurnherr, J. M. Toole, and A. J. Watson, 2012: Turbulence and diapycnal mixing in Drake Passage. J. Phys. Oceanogr., 42, 2143-2152, https://doi.org/10.1175/JPO-D-12-027.1.

Talley, L. D., 2013: Closure of the global overturning circulation through the Indian, Pacific, and Southern Oceans: Schematics and transports. Oceanography, 26 (1), 80-97, https://doi.org/ 10.5670/oceanog.2013.07.

Taylor, J. R., and R. Ferrari, 2010: Buoyancy and wind-driven convection at mixed layer density fronts. J. Phys. Oceanogr., 40, 1222-1242, https://doi.org/10.1175/2010JPO4365.1.

Thompson, L., and G. C. Johnson, 1996: Abyssal currents generated by diffusion and geothermal heating over rises. Deep-Sea Res. I, $\mathbf{4 3}$, 193-211, https://doi.org/10.1016/0967-0637(96)00095-7.

Thorpe, S. A., 1987: Current and temperature variability on the continental slope. Philos. Trans. Roy. Soc. London, 323A, 471-517, https://doi.org/10.1098/rsta.1987.0100.

Thurnherr, A. M., and K. G. Speer, 2003: Boundary mixing and topographic blocking on the Mid-Atlantic Ridge in the South Atlantic. J. Phys. Oceanogr., 33, 848-862, https://doi.org/ 10.1175/1520-0485(2003)33<848:BMATBO>2.0.CO;2.

_ L. L. C. St. Laurent, J. M. Toole, and J. R. Ledwell, 2005: Mixing associated with sills in a canyon on the midocean ridge flank. J. Phys. Oceanogr., 35, 1370-1381, https://doi.org/10.1175/ JPO2773.1.

Toole, J. M., 2007: Temporal characteristics of abyssal finescale motions above rough bathymetry. J. Phys. Oceanogr., 37, 409427, https://doi.org/10.1175/JPO2988.1.

Townsend, A. A., 1976: The Structure of Turbulent Shear Flow. 2nd ed. Cambridge University Press, 429 pp.

Vallis, G. K., 2017: Atmospheric and Oceanic Fluid Dynamics. 2nd ed. Cambridge University Press, 964 pp.

Waterhouse, A. F., and Coauthors, 2014: Global patterns of diapycnal mixing from measurements of the turbulent dissipation rate. J. Phys. Oceanogr., 44, 1854-1872, https:// doi.org/10.1175/JPO-D-13-0104.1.

Wenegrat, J. O., J. Callies, and L. N. Thomas, 2018: Submesoscale baroclinic instability in the bottom boundary layer. J. Phys. Oceanogr., https://doi.org/10.1175/JPO-D-17-0264.1, in press.

Wunsch, C., 1970: On oceanic boundary mixing. Deep-Sea Res. Oceanogr. Abstr., 17, 293-301, https://doi.org/10.1016/ 0011-7471(70)90022-7.

Young, W. R., 2012: An exact thickness-weighted average formulation of the Boussinesq equations. J. Phys. Oceanogr., 42, 692-707, https://doi.org/10.1175/JPO-D-11-0102.1. 